\title{
Another face of dermal fillers
}

\author{
Henryk Witmanowski ${ }^{1}$, Katarzyna Błochowiak ${ }^{2}$
}

${ }^{1}$ Department of Plastic, Reconstructive and Aesthetic Surgery, Nicolaus Copernicus University in Torun, Collegium Medicum in Bydgoszcz, Poland

2Department of Oral Surgery and Periodontology, Poznan University of Medical Sciences, Poznan, Poland

Adv Dermatol Allergol 2020; XXXVII (5): 651-659

DOI: https://doi.org/10.5114/ada.2019.82859

\begin{abstract}
Potential complications related to dermal fillers depend on the type of the fillers and the site of their application. The classification of dermal filler complications can be divided into early and late, and into minor and major events. According to their mechanism, they can be divided into non-ischaemic and ischaemic complications. The aim of this study is to present possible complications related to dermal fillers, to explain their aetiology and to propose preventive management and treatment. Non-ischaemic complications can be technique-dependent and include the reactions at the site of the injection, contour irregularities, and inflammatory and infectious reactions. They are usually resolved as a matter of course after 2-7 days. Arterial or venous occlusion leads to ischemia, with subsequent necrosis of the skin and/or vision loss.
\end{abstract}

Key words: dermal fillers, plastic surgery, hyaluronic acid, collagen, side effects, poly-L-lactic acid.

\section{Introduction}

Facial fillers provide adequate volume on depressed areas of the skin and allow a youthful, rejuvenated appearance to be maintained. Soft tissue augmentation is increasing in popularity as a result of growing interest in facial contouring, breast augmentation and cheek reshaping. In aesthetic and reconstructive surgery, facial soft tissue augmentation is performed using several biomaterials [1]. Dermal fillers can be presented as fluids, biological fragments or suspensions of particles or microspheres, each with a specific implantation method and possible complications. Dermal fillers are classified as temporary or biodegradable; permanent or non-biodegradable; and a combination of both materials [2]. Softtissue fillers are classified by the duration of their effect as temporary, long-lasting, semi-permanent, and permanent ones. Examples of biodegradable fillers include hyaluronic acid (HA), collagen (bovine, porcine, and human), poly-L-lactic acid (PLLA), calcium hydroxylapatite (CaHA), and dextran beads in hyaluronic acid. Examples of nonbiodegradable fillers include polymethylmethacrylate microspheres with bovine collagen, polymethylmethacrylate microspheres suspended in carboxygluconate gel, silicone, saturated hydrocarbons, polymethylmethacrylate silicone suspension, acrylic hydrogel particles suspended in hyaluronic acid, polyacrylamide gel, polyvinyl microspheres suspended in polyacrylamide, e-polytetrafluoroethylene, Gore-tex, and autologous fat [3]. Treatment with fillers is regarded as effective and safe. However, complications can arise and education, recognition and early treatment are of utmost importance. Potential complications related to dermal fillers depend on the type of the filler and the site of their application. Complications associated with temporary fillers usually occur immediately after the injection and are easily treated, or, alternatively, they resolve spontaneously. Complications with permanent fillers may occur years after implantation and typically require more complex treatment [2]. The classification of dermal filler complications can be divided into early and late and into minor and major events. According to the time of onset, the filler complications can be classified as immediate onset (up to $24 \mathrm{~h}$ after the procedure); early onset ( $24 \mathrm{~h}$ to 4 weeks); and delayed onset (more than 4 weeks) [4]. According to their mechanism, they can be divided into non-ischaemic and ischaemic complications.

The aim of this study is to present possible complications related to dermal fillers, to explain their aetiology and to propose preventive management and treatment.

Address for correspondence: Katarzyna Błochowiak DDS, PhD, Department of Oral Surgery and Periodontology, Poznan University of Medical Sciences, 70 Bukowska St, 60-812, Poznan, Poland, e-mail: kasia@naszdentysta.com.pl Received: 29.12.2018, accepted: 30.01.2019. 


\section{Non-ischaemic complications}

Non-ischaemic complications are not specific to the type of dermal fillers but they can be techniquedependent. Non-ischaemic complications include a wide spectrum of adverse effects of reactions at the injection site, such as erythema, swelling, itching, bruising and induration that are very common in the first $72 \mathrm{~h}$ after implantation. Another group of non-ischaemic complications consists of persistent erythema, itching, depigmentation, induration, lumpiness, persistent granulomatous foreign body reaction, ulceration, contour irregularities, bluish-discoloration, the Tyndall effect, inflammatory reactions, and infection or biofilm formation. Ecchymosis and haematomas can occur if the patient ingests alcohol, anti-platelet-aggregating drugs, ticlopidine, vitamin E or non-steroidal anti-inflammatory drugs (NSAID) days before or after implantation. Such complications are usually resolved as a matter of course after 2-7 days. They are non-specific to the type of fillers [2]. Bruising may be treated with cold compresses after the procedure, arnica, aloe vera, or vitamin K creams [4]. Hematoma and ecchymosis have been reported in $5 \%$ of autologous fat transfers [5]. Swelling can occur about 1 week after implantation. If the swelling is significant or persistent, it should raise concerns about lymphatic obstruction, a sterile abscess or hypersensitivity reaction [6]. Lymphatic obstruction can present as malar oedema after tear trough correction. This complication is most likely to be multifactorial and related to the patient's degree of preprocedural lymphatic obstruction, the depth of the injection within the suborbicularis oculi fat pad, the volume injected, and the viscosity of the filler used [6]. The incidence of malar oedema after injection of HA into the periorbital region is estimated at $2 \%$ [7]. Transient oedema and bruising are expected after injection and can be minimised by avoiding blood thinners 2 weeks prior to injection, applying numbing cream with a vasoconstrictor such as epinephrine prior to injection, and the use of cool compresses after the injection. Itching and erythema are often treated with topical or intradermal applications of corticosteroids. Persistent erythema is associated with several different facial fillers and can be prevented by avoiding alcohol consumption, sun exposure and exercise during the first days following the procedure. Some practitioners recommend oral, intralesional or topical administration of corticosteroids in all site injection side effects [2]. Some transient swelling in the immediate postprocedural period occurs with all dermal fillers, but may vary in timing and severity depending on the specific product used. The patient factors, such as dermographism, may also influence the amount of swelling [4]. The treatment includes NSAIDs and anti-inflammatory enzymes such as Wobenzym Vital or Bromelain in mild and moderate swelling or steroids in severe swelling [4]. All dermal fillers can induce scar formation especially in predisposing patients. Scars have been reported in $2 \%$ of autologous fat transfers [5]. Hypertrophic scarring is treated with triamcinolone injections and a pulsed dye laser.

Another possible complication is infection. There are two possible routes of infection: bacterial contamination at the site of injection and a bacterial infection and inflammatory response to the dermal filler. To minimise infection, thorough sterilisation of the treatment site should be performed prior to injection. The biocompatible nature of non-absorbable hydrogel polymers is thought to predispose them to bacterial contamination, biofilm formation, and infections with potential secondary abscess formation [8]. Early infections can occur between several days and a week after the injection, whereas late infections occur from several weeks up to months after the injection. Lesions of early infection can be almost indistinguishable from an inflammatory hypersensitivity response. They may resolve spontaneously or require minimal medical interference. Infections can manifest as granulomatous allergic tissue reactions developing into abscesses, localized granulomatous tissue reactions, abscess-like nodules, delayed granulomatous reactions, sterile abscesses, foreign body nodules, or delayed onset reactions [9]. When the bacterial culture is positive, skin flora such as staphylococcus epidermidis or aureus predominate. All infections can turn into an abscess, which is defined as fluctuating swelling with redness and/or tenderness or pain around the injection site. According to Kadouch et al., polyalkylimide gel fillers predispose to abscess formation [8]. Abscesses and their surgical treatment may result in scar formation. Aseptic abscess and cyst formation is another rare adverse localized reaction that can occur after the filler. The abscess is technically "sterile," meaning negative culture, and can be confined to the injection site or extend to adjacent tissue. Histologically, there are numerous neutrophils, lymphocytes, plasma cells, and multinucleated giant cells surrounding particles of injected collagen, cellular debris, and haemorrhage [9]. In addition to abscesses, infections can manifest as single or multiple erythematous nodules or infectious granulomas. The infectious granuloma is characterized by suppurative or caseating granuloma with central caseation necrosis and prominent neutrophilic infiltrate. The epidermis can present a variable degree of acanthosis, hyperkeratosis, and follicular hyperkeratosis [10]. Another early manifestation of infection is an erythematous or painful lump within the first few days after the injection. This infection is treated with a 7-day course of broad-spectrum antibiotics. A patient who presents with a painful nodule more than 2 weeks after the injection should be evaluated for atypical infection, such as mycobacteria, or a type IV hypersensitivity reaction, with a biopsy for both $\mathrm{H}+\mathrm{E}$ and culture. Intralesional corticosteroid injections or excision by means of a direct approach should be performed. When inflammatory features are present and infection cannot 
be ruled out, oral antibiotics should be administered. All abscesses or recurrent low-grade inflammations should be treated surgically [8]. The routine treatment includes systemic administration of antibiotics and close followup. Another possible complication related to HA is a biofilm. According to a new concept, many HA complications are due to biofilms and not due to allergies or another inflammatory response. Biofilms are groups of microorganisms in which cells stick to each other in a three-dimensional structure on a given surface. Usually the biofilm is covered by a polymeric substance which offers antibiotic resistance. Biofilms live in a dormant state (low-grade infection), but an active infection can be triggered by trauma, hematogenic infection or iatrogenic manipulation. Because the biofilms are hard to culture and to detect, many HA complications are not correctly diagnosed and are attributed to allergies [11]. There are five steps in biofilm formation: initial attachment, irreversible attachment, maturation I, maturation II and dispersion. In the dispersion phase in particular, the biofilm secretes a protective barrier consisting of carbohydrates. This barrier is increasingly antibiotic-resistant [11]. In cases of the suspected biofilm, more intense antibiotic regimens should be considered, and a potential consultation with an infectious disease specialist. Biofilms have impaired immune system penetration, reduced growth rates and susceptibility, and produce substances that individual bacteria are unable to produce alone. Biofilms excrete a protective and adhesive matrix, which may interfere with macrophage phagocytosis, and biofilms also allow for up to 1000 times greater resistance to antibiotics. As biofilms progress, they become more antibiotic- and culture-resistant [9]. Biofilms tend to have more of an indolent inflammation and the causative bacteria can be elusive. When symptoms of infection such as pain, redness, swelling, heat at the site or pus and fistula appear late, even months after the injection of HA, activation of a dormant biofilm infection is to be considered [11]. The identification of biofilms is difficult. It is usually the case that microbiological cultures from biofilm-infected tissue are negative. Diagnostic methods include classic culture plates, the "tube method", the Congo red agar test, and in situ fluorescent hybridization (FISH) [11]. Treatment involves culturing and draining any fluctuant areas and immediately starting any systemic antibiotics, even if the culture is negative [9]. Only after a trial of antibiotics should intralesional high-dose steroids be considered, and if all else fails, excision of the filler can help eradicate the biofilm [9]. Broad-spectrum antibiotics like ciprofloxacin, amoxicillin or clarithromycin should be used for up to 6 weeks. All permanent fillers predispose to biofilm formation. Another important therapeutical measure is the use of hyaluronidase (HAG) to remove the HA. Lyses of HA with HAG destroy the mechanical support of the biofilm colony. In cases of both inflammation and granuloma formation related to hyaluronic acid injection, HAG can be used to dissolve the HA filler product. HAG prevents further foreign body reactions, but is not recommended in cases of active infection, because it could spread the infection. Alternative therapies include steroids, NSAIDs, antihistamines and 5-flouracil. 5-Flouracil reduces the biofilm through inhibition of DNA synthesis in bacteria. On the other hand, steroids, NSAIDs and antihistamines can exacerbate an infection. Dermal fillers trigger recurrent herpetic lesions and antiviral prophylaxis is recommended in case of lip augmentation. They are contraindicated in the presence of active herpetic lesions [2].

Some early complications include temporary discoloration of the skin in or around the injection site, resulting in redness, whiteness, or hyperpigmentation. The Tyndall effect is a technique-dependant complication related to HA-based and collagen fillers. It involves a slight blue-gray discoloration at the site of the filler injection. It occurs if the filler is placed too superficially. High-risk areas for this Tyndall effect include the nasojugal folds, nasal dorsum, the lip, the infraorbital troughs, and fine superficial perioral and periorbital lines [9]. The Tyndall effect is defined as the scattering of light when it encounters a turbid medium. It is best avoided by injecting filler products at the proper depth within the tissue. Once present, the discoloration can be addressed with an injection of HAG to degrade the filler [6]. In cases of hyperpigmentation, the first therapeutic approach should be with a bleaching agent such as topical hydroquinone (2-8\%) and Retin-A (tretinoin) combined with daily fullspectrum sunscreen application. In cases of unresponsive hyperpigmentation, chemical peels may also be used. If the treatment is not successful, the next steps include the treatment with intense pulsed light, a pulsed dye laser, or fractional laser [4].

One of the most common side effects of dermal fillers is nodule and granuloma formation. A nodule is defined as a firm swelling or induration lacking the clinical features of inflammation, such as erythema, oedema, heat, and tenderness or pain. This complication is reported in HA fillers, CaHA, PLLA, collagen-based fillers, and in silicone- and polyacrylamide-based fillers. All these materials can potentially induce nodules, lumps and granulomas. However, HA fillers are safer due to their temporary nature and response to HAG injections. Palpable nodules can occur due to an asymmetric or irregular placement of the filler by agglomeration of the microspheres and also due to superficial implantation [2]. Palpable, but not visible, nodules from CaHA are common and typically resolve anywhere from 2 to 6 weeks after placement. They can also be injected with normal saline to dilute the product and make it more malleable, allowing the physician to redistribute the product with massage. If the nodules are cosmetically apparent or painful, the recommended treatment includes the intralesional injection of steroids or surgical excision. The nodules from PLLA are 
more likely to be fibrotic [6]. O'Daniel recommends treatment of recurrent facial nodules after PLLA with oral corticosteroids and periodic intralesional steroid injections [12]. Intralesional steroid injections have to be repeated to establish the right dose [13]. The nodules from other fillers should be removed surgically rather than treated with an intralesional injection of corticosteroids, using micro-electric dissection, punch excision and dermabrasion [2]. Visible hard nodules or hard granuloma in the subcutaneous fat are an indication for surgical excision [13]. The long-term side effects of semi-permanent fillers which are a combination of biodegradable materials like HA with non-biodegradable materials like acrylic hydrogel particles include skin indurations including nodules and granulomas with or without fistulation [14]. All dermal fillers induce at least a mild foreign body reaction. The histological appearance of the granuloma is specific to each type of the filler. They are presented as microspheres that are distant from each other and surrounded by macrophages, giant cells, fibroblasts and extensive collagen fibres. The lesions are characterized by patchy distribution and a non-suppurative pattern. The infiltrate is composed mainly of epithelioid and foamy histiocytes, lymphocytes, plasma cells, eosinophils and rarely neutrophils [10]. Foreign-body granulomas which subsequently form an infectious ulcer are a well-documented side effect of the polyacrylamide filler [1]. Disseminated and recurrent sarcoid-like granulomatous panniculitis has also been described as a very rare reaction to bovine collagen injection [9]. Factors which may influence granuloma development include properties of the filler, volume injected, and previous infections or trauma. The shape of the methacrylate microspheres, which are comparatively irregular and sharp-edged structures in permanent and semi-permanent fillers, may be an important factor in inducing a more severe granulomatous reaction [14]. The polymethylmethacrylate fillers cause granuloma about 6-24 months after implantation [2]. For most dermal fillers, the time interval between implantation and the onset of symptoms ranges from 2 months to 6 years [2]. On the other hand, autologous fat transfers do not cause granuloma [5]. Treatment of granulomas includes intralesional steroids, 5-fluorouracil, anti-inflammatory and immunomodulatory drugs like minocycline, rifampicin or hydroxychloroquine. Granulomas often respond to the intralesional injection of a mixture of 5 -fluorouracil (250 mg/ml), triamcinolone acetonide $(10 \mathrm{mg} / \mathrm{ml})$ plus mepivacaine $(1 \mathrm{ml})$, which is given first twice a week and then once a week; in addition to allopurinol given at a dose of 300-600 mg/day [15]. An alternative option is the use of NSAIDs, antihistamines and tacrolimus. In the case of widespread lesions or repeated failure of conservative therapies, surgical excision is the treatment of choice. Surgical extirpation can also allow a dermatologist to prevent the cutaneous side effects of intradermal steroid or 5-fluorouracil injection. A histopathological confirmation is recommended before undertaking a surgical excision of the granuloma [14]. Granulomas can develop even many years after injections of permanent fillers and it is important to have a long-term follow-up for patients injected with permanent and semi-permanent fillers. In patients with a history of inflammatory reactions, further injections with filler products should be performed with caution. Non-absorbable hydrogels can induce a limited inflammatory reaction and thin fibrous capsules after the injection, thus exhibiting migratory potential [8]. The migration of implanted material is difficult to solve because the particles are encapsulated by collagen fibres and can present as nodules or indurations that will require surgical removal. This complication may appear even many years after implantation [2]. The pathogenesis of filler migration is explained by gravity, muscle movement, and post-operative massage of the injected material. The practitioner's skill is also important [16]. Jordan and Stoica report a few possible mechanisms of filler migration: intravascular injection, lymphatic spread, antigravity, gravity, pressure-induced displacement, muscle activity, massage, or an improper injection technique [3]. On examination, the lesion may resemble a neoplasm or granulomatous disease that presents months to years after treatment. The filler migration itself usually does not require treatment or surgery. Permanent fillers composed of solid particles have a reduced tendency to migrate [17]. Filler migration from the forehead, glabellar and nose to the eyelid is the most common [16]. Since filler migration is mostly caused by permanent fillers, surgical excision is the recommended choice of treatment. Intralesional injection of steroids may be considered as an alternative treatment [16].

Dermal fillers are composed of different materials. These materials have different biocompatibility, biodegradability, and immunogenicity and can trigger divergent anaphylactic reactions from local angioedema and rash at the injection site to generalized or systemic manifestations or even anaphylactic shock. Hypersensitivity reactions can result from proteins, impurities or the chemical agents used in the cross-linking process, residual byproducts of bacterial fermentation, and the size and smoothness of the filler particles [4]. The rates of hypersensitivity vary among different products and dermal fillers. Generally, the risk of hypersensitivity to HA, CaHA, and PLLA is low [5]. The incidence of the hypersensitivity reaction to injectable $\mathrm{HA}$ is estimated at $0.02 \%$ [5]. Different dermal fillers can predispose to one particular anaphylactic reaction. Hypersensitivity reactions to bovine collagen and polymethylmethacrylate typically present as late granulomas [2]. One of the advantages of autologous fat transfer over other facial fillers in both early and late events is the absence of hypersensitivity reactions [6]. Hypersensitivity reactions can be classified as acute or delayed, depending on the time of onset. Some practitioners divide hypersensitivity 
reactions into early (< 14 days), late (14 days -1 year), and delayed ( $>1$ year) [5]. There are two main types of hypersensitivity reactions. Type I hypersensitivity reactions occur within minutes or hours of injections, due to an immunoglobulin E (IgE)-mediated immune response to the dermal filler. They may manifest as angioedema or anaphylactic reactions occurring after initial or repeated exposure. A more systemic reaction can occur 48 to $72 \mathrm{~h}$ after the injection, and is characterized by fever, malaise and urticaria, which can be treated with short-term oral steroids. Other more rare systemic complications, such as flu-like symptoms, paraesthesias or difficulty breathing, have also been described after bovine collagen injection [9]. Delayed hypersensitivity reactions include induration, erythema, and oedema and are initiated by T lymphocytes and mediated by CD4+ cells. This is type IV hypersensitivity. The rates of delayed hypersensitivity vary between $0.02 \%$ and $4 \%$ [18]. Approximately $3 \%$ of the general population are allergic to bovine collagen and $70 \%$ of these reactions manifest as a delayed type IV hypersensitivity reaction 48 to $72 \mathrm{~h}$ after the first test [9]. These typically occur 48-72 h after the injection, but may be seen as late as several weeks post-injection and may persist for many months. The aetiology of delayed hypersensitivity is unknown, but there are a few potentially influencing factors, including previous infections and trauma, the injection technique, filler volume, repeated treatments, intramuscular implantation and different properties of the filler [18]. The most important issue is to properly distinguish infectious causes from immune-mediated causes of delayed hypersensitivity, because if an infection is suspected, steroids should not be prescribed. Differential diagnosis is difficult because hypersensitive reactions manifesting as persistent facial oedema can be accompanied by inflammatory nodules or foreign body granulomas. Furthermore, their outbreak can be triggered by a flu-like illness. In immune-mediated origins, the injection area is asymptomatic for months between the last treatment and reaction onset, symptoms occur simultaneously and patients are systemically well. In the event of infection, symptoms are localized or restricted to a discrete area and there is usually a need for an empirical antibiotic treatment [18]. The type IV hypersensitivity reactions are unresponsive to antihistamines. Steroids and HAG are effective in type IV lesions of hypersensitivity [18]. Type I local anaphylactic lesions can be treated with topical steroids or immunosuppressants, but in severe cases, systematic treatment is required. In addition to steroids, topical tacrolimus ointment can be applied. Anaphylactic reactions require immediate attention and a prompt response, because a small number of patients may develop life-threatening anaphylactic shock. First-aid kits including routine adrenaline shots, steroids and a tracheotomy kit are vital and should always be accessible.

Another possible complication related to autologous fat transfer is fat necrosis and oil cysts. Their rates are estimated at $2 \%$. Irregular fat distribution is treated as an undesirable event and occurs at 2\% [6]. Another possible complication related to the perioral area is the formation of extravasation cysts such as mucocele. If small salivary glands are injected, retention cysts and sialadenitis can occur, but this has not yet been described using resolvable HA [17].

\section{Ischaemic and visual complications}

The most severe and feared early complication associated with the use of dermal fillers and volume enhancers is arterial or venous occlusion, which leads to ischaemia, with subsequent necrosis of the skin and/or vision loss. Necrosis can be attributed to one of two factors - an interruption of vascular supply due to compression or frank obstruction of vessels by a direct injection of the material into a vessel itself [19]. Occlusion results from the direct intravascular injection of the product, vascular injury, or external compression of the blood supply by the surrounding filler/volume enhancer material or swelling [19]. The incidence of vascular occlusion has been reported as being up to 3 in 1000 injections. For $\mathrm{HA}$ injections, the incidence of vascular occlusion may be slightly less at 3-9 per 10,000 injections. The most high-risk areas include the glabellae and nasal area, but can also occur in the lip, nasolabial fold and temple. They are technique-dependant complications. The mechanism of action of filler-associated ischaemia is due to direct intra-arterial injection of the product. Early recognition of vascular occlusion and swift and aggressive treatment are required to avoid any irreversible changes. However, initial symptoms of a vascular event are often dismissed as simple postprocedural discomfort [20]. Signs of soft tissue ischaemia include whitening or blanching on the injection, pain, mottling, blister formation, bluish discoloration and later, tissue necrosis. Not all of these signs may be present. The whitening or blanching and pain may be transient and unnoticed if anaesthetics are often administered concurrently. Mottling in the area of a vascular distribution larger than the injected area is a clue that vascular ischaemia is occurring. The mottled appearance can then change into bluish discoloration [21]. On the other hand, blister formation can be confused with a herpetic infection. Once the deep dermal layers are affected, scarring is likely to occur. Treatment of skin necrosis is based on skin grafts, local flaps, surgical debridement, and dressings with different materials. However, these treatments often result in unsightly skin loss, scarring, and asymmetry. Stem cells promote angiogenic processes and wound healing by secreting angiogenic factors, differentiating into different cells contributing to neovascular formation and stimulating cells that perform significant roles in wound healing [22]. Sung et al. reported the use of adipose-derived stem cells for skin necrosis and acute inflammatory reaction after filler 
injections for soft tissue augmentation [22, 23]. Adipose tissues are harvested from the patients' abdomens by liposuction, centrifuges and mixed with collagenase type $\mathrm{II}$ and liquefied by saline in a syringe [22].

Visual complications related to dermal fillers in the periorbital area include vision loss, decreased eye movement or ophthalmoplegia, droopy eyelid or ptosis and cerebral infarct. Other initial symptoms are orbital pain, dizziness, pupil dilatation, diplopia, and partial retinal ischaemia on fundoscopy [24]. Most cases of blindness are caused by autologous fat and HA injections. Nearly half of the cases of filler-related blindness reported thus far are attributable to fat transfers. The cases of blind ness after the cosmetic injection following autologous fat transfer are more likely to be attributable to intravascular embolism rather than external pressure on the vessel [17]. Myung et al. classified periocular complications related to blindness following HA fillers into type (blindness without ptosis or ophthalmoplegia), type II (blindness and ptosis without ophthalmoplegia), type III (blindness and ophthalmoplegia without ptosis), and type IV (blindness with ptosis and ophthalmoplegia) [25] Improved visual acuity in patients with vascular occlusion after a filler injection is extremely rare. By contrast, periocular symptoms such as ptosis and ophthalmoplegia recover dramatically during the follow-up period. Almost all patients experienced total recovery of ptosis and ophthalmoplegia except for one type IV patient with persistent mild strabismus [25]. Thrombosis is related to the properties of the filler material, such as molecular weight or size. The most high-risk injection areas associated with visual compromise include the glabellae, forehead, nasal region, nasolabial folds and temple. The arteries in these high-risk areas have direct communication with the ophthalmic artery. If embolization with an HA filler into the retinal artery is suspected, the early retrobulbar injection of 2 to $4 \mathrm{ml}$ of highly concentrated HAG is recommended in order to decomplex both intravascular and interstitial HA [17]. Vascular thrombosis most commonly occurs in facial arteries such as supratrochlear arteries and angular arteries, which may lead to skin necrosis of the area between the eyebrows and nose. Ocular arteries and intracranial arteries may also be affected, leading to tissue deactivation, blindness and paralysis. If thrombosis occurs in superficial arteries, patients will experience sharp pain and geographic pale skin, or delayed onset of dull pain with cyanotic skin, which later evolves into redness, pus, scabs, and eventually, scar tissue [26]. There is also a retrograde mechanism of vascular occlusion. Chou et al. postulate that the calcium hydroxyapatite ( $\mathrm{CaHA})$ emboli can migrate via the dorsonasal artery back to the main ciliary arteries and occlude the short posterior ciliary arteries, which supply the superior nasal choroid and the optic nerve. In cases of vascular thrombosis practitioners should stop the injection immediately and extract some filler material if possible. Administration of hot packs to the affected sites and massage are recommended. Systemic treatment usually takes place in the hospital with the patient being carefully monitored, as with all potent vasoactive drugs. Even if presentation is delayed in the patient, treatment is still recommended, because it may restore normal circulation and speed up the healing process, as was the case with the authors' patients. In the case of HA use, the first step and gold standard is HAG injection. HAG is a soluble and naturally occurring protein enzyme that hydrolyzes both natural and cross-linked HA dermal fillers. The HAG is very important for managing cutaneous complications secondary to intravascular HA filler injection. The dose of HAG is estimated depending on the number of adjoining areas affected [27]. The HAG is generally well tolerated; the most common complication is injection site reaction. In general, human recombinant HAG is more recommended than the animal-derived products because of a lower hypersensitivity risk [6]. Expert recommendations for HAG dosing and indications can vary significantly. HAG may be injected directly into the affected site, with doses of $40 \mathrm{U}(0.1 \mathrm{ml})$ or more per $\mathrm{cm}^{2}$, after which it starts to dissolve the material immediately and lasts for between 24 and $48 \mathrm{~h}$ [19]. The total dose of HAG can be modified according to the improvement. The initial dose of approximately 300 units (U) of HAG can be increased to $1,000 \cup$ to achieve reperfusion when lower doses are inadequate [6]. Fang et al. recommend a dose of a minimum of 200-300 $\mathrm{U}$ of HAG and up to 1,500 U [27]. The recent consensus recommendations advocate immediate administration of $200 \mathrm{U}$ of HAG in cases of impending necrosis. Another recommendation is a local HAG injection of $150 \mathrm{U}$ HAG to dissolve $1 \mathrm{ml} \mathrm{HA}$. The optimal time of injection in the case of HA vascular complications has not been determined, but it is wise to administer it as early as possible. Furthermore, HAG can be used, regardless of the type of the filler used. It has oedema-reducing effects and contributes to a reduction in proinflammatory cytokines and growth factors. The polyphasic fillers degrade at a much lower dose of HAG than monophasic fillers and it is hypothesized that this is due to the higher degree of cross-linking in polyphasic fillers and thus the greater amount of exposed surface area on which the HAG can act. Moreover, patients should take $300 \mathrm{mg}$ aspirin orally. Intravenous administration of $20 \mathrm{mg}$ dexamethasone and local or systemic vasodilators such as nitroglycerin decrease thrombosis involvement. Therapy also includes anticoagulants like low-molecular-weight heparin. Bruna et al. also recommend Prostaglandin E1 (PGE1) to promote vasodilation. Furthermore, localized skin breakdown should be treated with topical (with or without systemic) antibiotics and antivirals, especially if necrosis is around the mouth [19]. Vascular thrombosis causes local blood supply impairment and increases the risk of bacterial infection. Redness, ulcers and pustules result from secondary infection from poor blood supply 
after vascular thrombosis. These lesions can be treated by intravenous administration of $20 \mathrm{mg}$ dexamethasone once a day and hyperbaric oxygen therapy ( $\mathrm{HBO} 2$ ) once a day for 6 sessions. Additionally, topical recombinant human epidermal growth factor gel on the lesions for 10 days and systemic administration of antibiotics are recommended. HBO2 improves blood supply, prompts revascularization and fights anaerobic causative bacteria [26]. Furthermore, $\mathrm{HBO} 2$ is thought to be efficacious in these situations due to a variety of mechanisms: oxygenation of ischaemic tissues, reduction of oedema, amelioration of ischaemic/reperfusion injury, and promotion of angiogenesis and collagen maturation. $\mathrm{HBO} 2$ is treated as adjuvant therapy. The recommended scheme of this therapy consists of 6 total treatments, performed twice daily, the initial 2 at $3.0 \mathrm{~atm}$ absolute for $90 \mathrm{~min}$ followed by 4 treatments at $2.4 \mathrm{~atm}$ for $90 \mathrm{~min}$, all with air breaks every $30 \mathrm{~min}$. The first cycle should be begun within $15 \mathrm{~h}$ after the initial injection [28]. If necrosis has occurred, it is important to minimize scarring by providing careful wound care with daily dressings. The wound should be covered with ointment to prevent crusting and to keep out bacteria until healing is complete. Treatment of the resulting scar involves silicone pads and an intralesional steroid injection. If a scar remains, it may be treated with light dermabrasion, surgical revision, or an injection with a filler to restore the contour. A minimum of 3 months should be allowed for scar maturation and establishment of collateral circulation before surgical attempts at scar revision [19]. In the treatment of ischaemic skin, time is the most prognostic factor. The treatment should be initiated no later than 3 days after the procedure [20].

\section{Prevention of dermal filler complications}

All complications can be caused by three categories of factors: patient-related factors, product-related factors and procedure-related factors. Taking a thorough history of skin conditions, allergies, systemic disease, current medication, and previous procedures is mandatory and allows to avoid severe postoperative complications. Several skin and systemic diseases are contraindications for the treatment with dermal fillers. The inflammatory and infective conditions that decrease the skin barrier function can trigger the postoperative skin infection or the biofilm formation. The treatment of inflammatory conditions such as acne, rosacea, and dermatitis is important as it is allowing adequate time for the restoration of the barrier function. This may require 3-4 weeks after apparent clearance [29]. Moreover, the remote infections of the urinary tract, sinuses, intestinal tract, and oral cavity, and a history of herpes simplex infection in the intended injection area decrease the risk of the formation of late-onset nodules. Filler treatments are contraindicated in active autoimmune diseases such as systemic lupus erythematosus, rheumatoid arthritis, mixed connective tissue disease, and Hashimoto's thyroiditis [29]. The aseptic technique is mandatory and decreases the risk of injection-related infections. The chlorhexidine, chloroxylenol, iodophors, alcohol, and iodine are recommended topical antiseptics [29]. Although the injected material may have an important role in the occurrence of complications, most of them are technique-dependant ones. The common technical errors leading to filler complications include improper volume (too much or too little), improper depth (superficial or deep), wrong location (unfavourable or incorrect anatomic location), and inappropriate material $[10,30]$. Adverse events such as bruising or thrombosis can be avoided by ultrasonographic visualization of vessels before injection and during injection control and thus early counter-action of thrombosis or compression of structures is possible [17]. The exact location of injected fillers should be checked by Doppler ultrasound to avoid injury to arteries or veins [17]. There are some modifications to the technique to prevent further ischaemic complications. These include using local anaesthetics with epinephrine to vasoconstrict vessels prior to the injection, injecting small volumes per pass, aspiration prior to the injection, using low injection pressure, avoiding scarred areas and considering the use of blunt cannulas. For injection, if possible, we recommend a blunt cannula with an opening on the side of the cannula and injecting during the withdrawal of the cannula. On the other hand, practitioners should avoid using anaesthesia near a vascular bundle that may induce vascular spasm, such as those containing epinephrine, while also avoiding the use of epinephrine, so that the cause of blanching can be determined quickly [19]. In the event of arterial/venous occlusion and impending necrosis, the goal is to quickly promote increased blood flow to the affected area, so treatment should commence without delay, especially if visual access is affected. The filler injection should be stopped as soon as the patient complains of a toothache or headache. Moreover, nitroglycerin paste is recommended to facilitate more significant vasodilation, and low-molecular-weight heparin helps prevent thrombosis and embolism [24]. Each patient who receives HA filler therapy should be closely observed for $30 \mathrm{~min}$, and a few days' post-treatment follow-up is recommended. Some practitioners add paramethasone to the local anaesthetics to achieve vessel contraction, therefore lowering the risk of vessel punctures, but this modification causes pale skin and worsens the differentiation of anemization and thrombosis. However, none of techniques is 100\% effective in avoiding ischaemic complications. Another very important issue is the establishment of treatment indications with dermal fillers and the choice of appropriate material for soft tissue augmentation [14, 30-32]. Treatment with permanent and semi-permanent fillers is contraindicated in areas previously injected with other dermal fillers, in patients with a tendency to develop hy- 
pertrophic scars, in those with a history of autoimmune or inflammatory disease or receiving immunotherapy, and in patients with multiple allergies or those allergic to sodium hyaluronate. Permanent fillers require a longer follow-up. Injected filler materials and related complications should be examined by such techniques as ultrasonography, magnetic resonance imaging (MRI), and computed tomography [30-33]. High-frequency ultrasound imaging is helpful in distinguishing between granulomas and nodules-dermal filler deposits, which can form after dermal filler injections. Characteristic ultrasound features of granulomas include oval shape and blurred, irregular outer edges. Small hyperechoic areas are present inside the granulomas. The deposits after dermal fillers are anechogenic, with sharp, regular borders [34].

\section{Acknowledgments}

The study was conducted in the Department of Oral Surgery and Periodontology, Poznan University of Medical Sciences.

\section{Conflict of interest}

The authors declare no conflict of interest.

\section{References}

1. Park K, Nishiwaki F, Kabashima K, et al. A case of foreignbody granuloma of the glabella due to polyacrylamide filler and an intractable ulcer after skin biopsy: an immunohistochemical evaluation of inflammatory changes. Case Rep Dermatol 2013; 5: 181-5.

2. Medeiros CC, Cherubini K, Salum FG, et al. Complications after polymethylmethacrylate (PMMA) injections in the face: a literature review. Gerodontology 2014; 31: 245-50.

3. Jordan DR, Stoica B. Filler migration: a number of mechanisms to consider. Ophthalmic Plast Reconstr Surg 2015; 31 257-62.

4. Urdiales-Gálvez F, Escoda Delgado N, Figueiredo V, et al. Treatment of soft tissue filler complications: expert consensus recommendations. Aesthetic Plast Surg 2018; 42: 498-510.

5. Groen JW, Krastev TK, Hommes J, et al. Autologous fat transfer for facial rejuvenation: a systematic review on technique, efficacy, and satisfaction. Plast Reconstr Surg Glob Open 2017; 5: e1606.

6. Vanaman M, Fabi SG, Carruthers J. Complications in the cosmetic dermatology patient: a review and our experience (part 1). Dermatol Surg 2016; 42: 1-11.

7. Limongi RM, Tao J, Borba A, et al. Complications and management of polymethylmethacrylate (PMMA) injections to the midface. Aesthet Surg J 2016; 36: 132-5.

8. Kadouch JA, Tutein Nolthenius CJ, Kadouch DJ, et al. Complications after facial injections with permanent fillers: important limitations and considerations of MRI evaluation. Aesthet Surg J 2014; 34: 913-23.

9. Lucey P, Goldberg DJ. Complications of collagen fillers. Facial Plast Surg 2014; 30: 615-22.
10. El-Khalawany M, Fawzy S, Saied A, et al. Dermal filler complications: a clinicopathologic study with a spectrum of histologic reaction patterns. Ann Diagn Pathol 2015; 19: 10-5.

11. Dumitraşcu DI, Georgescu AV. The management of biofilm formation after hyaluronic acid gel filler injections: a review. Clujul Med 2013; 86: 192-5.

12. O'Daniel G. Management of late-onset, recurrent facial nodular reaction after poly-l-lactic (PLLA) Injections. J Drugs Dermatol 2017; 16: 1297-9.

13. Lemperle G, Rullan PP, Gauthier-Hazan N. Avoiding and treating dermal filler complications. Plast Reconstr Surg 2006; 118 (3 Suppl): 92S-107S.

14. Sachdev M, Anantheswar Y, Ashok B, et al. Facial granulomas secondary to injection of semi-permanent cosmetic dermal filler containing acrylic hydrogel particles. J Cutan Aesthet Surg 2010; 3: 162-6.

15. Haneke E. Managing complications of fillers: rare and notso-rare. J Cutan Aesthet Surg 2015; 8: 198-210.

16. Lee JI, Kang SJ, Sun H. Skin necrosis with oculomotor nerve palsy due to a hyaluronic acid filler injection. Arch Plast Surg 2017; 44: 340-3.

17. Vent J, Lefarth F, Massing T, et al. Do you know where your fillers go? An ultrastructural investigation of the lips. Clin Cosmet Investig Dermatol 2014; 7: 191-9.

18. Bhojani-Lynch T. Late-onset inflammatory response to hyaluronic acid dermal fillers. Plast Reconstr Surg Glob Open 2017; 5: e1532.

19. Souza Felix Bravo B, Klotz De Almeida Balassiano L, Roos Mariano Da Rocha C, et al. Delayed-type necrosis after softtissue augmentation with hyaluronic acid. J Clin Aesthet Dermatol 2015; 8: 42-7.

20. Hong JY, Seok J, Ahn GR, et al. Impending skin necrosis after dermal filler injection: a "golden time" for first-aid intervention. Dermatol Ther 2017; 30: e12440.

21. Chen Q, Liu Y, Fan D. Serious vascular complications after nonsurgical rhinoplasty: a case report. Plast Reconstr Surg Glob Open 2016; 4: e683.

22. Sung HM, Suh IS, Lee HB, et al. Case reports of adiposederived stem cell therapy for nasal skin necrosis after filler injection. Arch Plast Surg 2012; 39: 51-4.

23. Kim JH, Park SH, Lee BH, et al. Early intervention with highly condensed adipose-derived stem cells for complicated wounds following filler injections. Aesthetic Plast Surg 2016; 40: 428-34.

24. Lee SK, Kim SM, Cho SH, et al. Adverse reactions to injectable soft tissue fillers: memorable cases and their clinicopathological overview. J Cosmet Laser Ther 2015; 17: 102-8.

25. Myung Y, Yim S, Jeong JH, et al. The classification and prognosis of periocular complications related to blindness following cosmetic filler injection. Plast Reconstr Surg 2017; 140: 61-4.

26. Fan X, Dong M, Li T, et al. Two cases of adverse reactions of hyaluronic acid-based filler injections. Plast Reconstr Surg Glob Open 2016; 4: e1112.

27. Fang M, Rahman E, Kapoor KM. Managing complications of submental artery involvement after hyaluronic acid filler injection in chin region. Plast Reconstr Surg Glob Open 2018; 6: e1789.

28. Henderson R, Reilly DA, Cooper JS. Hyperbaric oxygen for ischemia due to injection of cosmetic fillers: case report and issues. Plast Reconstr Surg Glob Open 2018; 6: e1618.

29. Heydenrych I, Kapoor KM, De Boulle K, et al. A 10-point plan for avoiding hyaluronic acid dermal filler-related complications during facial aesthetic procedures and algorithms 
for management. Clin Cosmet Investig Dermatol 2018; 11: 603-11.

30. Hwang CJ. Periorbital injectables: understanding and avoiding complications. J Cutan Aesthet Surg 2016; 9: 73-9.

31. Chou CC, Chen HH, Tsai YY, et al. Choroid vascular occlusion and ischemic optic neuropathy after facial calcium hydroxyapatite injection - a case report. BMC Surg 2015; 15: 21.

32. Skrzypek E, Górnicka B, Skrzypek DM, Krzysztof MR. Granuloma as a complication of polycaprolactone-based dermal filler injection: ultrasound and histopathology studies. J Cosmet Laser Ther 2018; doi: 10.1080/14764172.2018.1461229.

33. Tal S, Maresky HS, Bryan T, et al. MRI in detecting facial cosmetic injectable fillers. Head Face Med 2016; 12: 27.

34. Mlosek RK, Skrzypek E, Skrzypek DM, Malinowska S. Highfrequency ultrasound-based differentiation between nodular dermal filler deposits and foreign body granulomas. Skin Res Technol 2018; 24: 417-22. 